\title{
DENSITY AND TEMPERATURE OF THE ELECTRON CORE IN THE INNER MAGNETOSPHERE OF SATURN FROM CASSINI/RPWS ANTENNAS
}

\author{
M. Moncuquet*, and E. Gkini*
}

\begin{abstract}
We study the large scale structures of the electron core in the inner magnetosphere of Saturn (from the G-ring to Rhea orbit - i.e. 2.8 to $9 \mathrm{R}_{\mathrm{s}}$ ). This study is deduced from the power spectra measurements acquired with the Cassini/RPWS electric dipole around the local plasma frequency (quasi-thermal noise spectroscopy), from July 2004 (SOI) to February 2010. We have especially investigated the radial diffusion and latitudinal confinement of the plasma torus towards Enceladus and Dione. We also discuss the longitudinal variations of the electron parameters using the recent SLS3 system. We will finally touch about the dust detection by using the RF power spectra measured on the monopole antenna and the possible effect of the dust on the observed electron parameters.
\end{abstract}

* LESIA, Observatoire de Paris, UMR CNRS 8109, 92195 Meudon, France 
